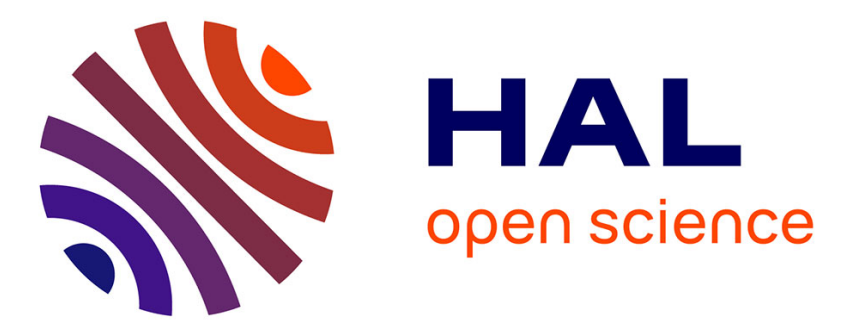

\title{
Understanding the effect of competition during evolutionary radiations: an integrated model of phenotypic and species diversification
}

Leandro Aristide, Hélène Morlon

\section{- To cite this version:}

Leandro Aristide, Hélène Morlon. Understanding the effect of competition during evolutionary radiations: an integrated model of phenotypic and species diversification. Ecology Letters, 2019, 10.1111/ele.13385 . hal-02408038

\section{HAL Id: hal-02408038 \\ https://hal.science/hal-02408038}

Submitted on 12 Dec 2019

HAL is a multi-disciplinary open access archive for the deposit and dissemination of scientific research documents, whether they are published or not. The documents may come from teaching and research institutions in France or abroad, or from public or private research centers.
L'archive ouverte pluridisciplinaire HAL, est destinée au dépôt et à la diffusion de documents scientifiques de niveau recherche, publiés ou non, émanant des établissements d'enseignement et de recherche français ou étrangers, des laboratoires publics ou privés. 


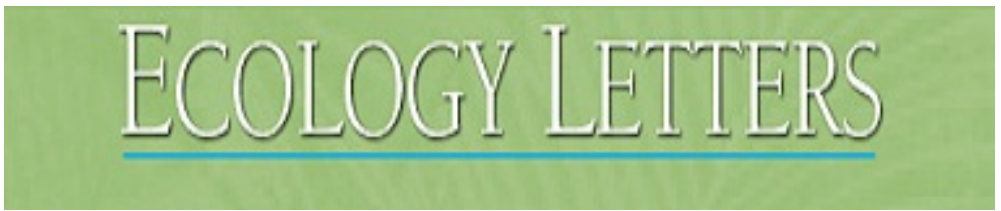

\section{Understanding the effect of competition during evolutionary radiations: an integrated model of phenotypic and species diversification}

\begin{tabular}{|c|c|}
\hline Journal: & Ecology Letters \\
\hline Manuscript ID & ELE-00459-2019.R2 \\
\hline Manuscript Type: & Letters \\
\hline $\begin{array}{l}\text { Date Submitted by the } \\
\text { Author: }\end{array}$ & 19-Aug-2019 \\
\hline Complete List of Authors: & $\begin{array}{l}\text { Aristide, Leandro; Ecole Normale Superieure, IBENS } \\
\text { Morlon, Helene; Ecole Normale Supérieure, IBENS }\end{array}$ \\
\hline Key Words: & $\begin{array}{l}\text { phylogenetics, macroevolution, disparity, diversity-dependence, } \\
\text { comparative methods, morphospace, protracted speciation, adaptive } \\
\text { radiation }\end{array}$ \\
\hline \multicolumn{2}{|c|}{$\begin{array}{l}\text { Note: The following files were submitted by the author for peer review, but cannot be converted to PDF } \\
\text { You must view these files (e.g. movies) online. }\end{array}$} \\
\hline
\end{tabular}




\section{TITLE:}

UNDERSTANDING THE EFFECT OF COMPETITION DURING EVOLUTIONARY RADIATIONS: AN INTEGRATED MODEL OF PHENOTYPIC AND SPECIES DIVERSIFICATION

AUTHORS: Leandro Aristide ${ }^{1,2}$ and Hélène Morlon ${ }^{1,3}$

AFFILIATION: ${ }^{1}$ École Normale Supérieure, Paris Sciences et Lettres (PSL) Research University, Institut de Biologie de l'École Normale Supérieure (IBENS), CNRS UMR 8197, INSERM U1024, 46 rue d’Ulm, F-75005 Paris, France ${ }^{2}$ leandroaristi@gmail.com; ${ }^{3}$ morlon@biologie.ens.fr

CONTACT INFORMATION: Leandro Aristide (leandroaristi@gmail.com). École Normale Supérieure, Paris Sciences et Lettres (PSL) Research University, Institut de Biologie de l'École Normale Supérieure (IBENS), CNRS UMR 8197, INSERM U1024, 46 rue d'Ulm, F-75005 Paris, France

STATEMENT OF AUTHORSHIP: LA \& HM designed the study. LA performed analyses. LA \& HM wrote the manuscript.

DATA ACCESIBILITY: R code for performing simulations under the described model is available at the RPANDA GitHub repository (https://github.com/hmorlon/PANDA).

RUNNING TITLE: Macroevolutionary dynamics under competition

KEYWORDS: adaptive radiation; phylogenetics; macroevolution; disparity; diversitydependence; comparative methods; morphospace; protracted speciation

TYPE: Letter

NUMBER OF WORDS: 150 (Abstract); 5000 (Main Text)

NUMBER OF REFERENCES: 80 
NUMBER OF FIGURES, TABLES, AND TEXT BOXES: 5 figures, 0 tables, 0 text boxes 


\section{INTRODUCTION}

A long-standing goal in ecology and evolutionary biology is to understand how competition among species modulates the origination and extinction of lineages, and the evolution of phenotypic differences among them (Sepkoski 1996; Doebeli \& Dieckmann 2000; Rundle \& Nosil 2005; Pfennig \& Pfennig 2009; Rabosky 2013; Vermeij 2013; Liow et al. 2015; Silvestro et al. 2015). Competition is in particular thought to play a key role during adaptive radiations - monophyletic lineages diversifying into several adaptive forms, where each occupies a portion of available ecological space through changes in relevant phenotypic traits (Simpson 1953; Futuyma 1998; Schluter 2000). Adaptive radiation theories have postulated that a lineage can diversify rapidly driven by competition and natural selection in face of an ecological opportunity, and that species and trait diversification rates should slowdown progressively as the available ecological space becomes more densely occupied (Gavrilets \& Losos 2009; Harmon et al. 2010; Rabosky 2013).

A large number of recent studies have looked for adaptive radiation signatures in comparative datasets (i.e. a phylogeny and associated trait data; Soulebeau et al. 2015). As phylogenetic methods that explicitly encapsulate ecological mechanisms are generally lacking (Pennell \& Harmon 2013; but see Drury et al. 2016; Clarke et al. 2017; Manceau et al. 2017), most researchers resort to pattern-based tests (e.g. $\gamma$ statistic for trees, Pybus \& Harvey 2000; Phillimore \& Price 2008; K statistics for traits, Blomberg et al. 2003) or phenomenological models (e.g. "early-burst" for traits, Harmon et al. 2010) built upon the general prediction for adaptive radiations of declining macroevolutionary rates. The most mechanistic studies have considered diversitydependent models built upon the prediction that rates decline as species accumulate (Rabosky \& Lovette 2008; Mahler et al. 2010; Etienne et al. 2012; Weir \& Mursleen 2013), or competition models where species phenotypes tend to evolve in unoccupied parts of phenotypic space (Drury et al. 2016; Clarke et al. 2017; Manceau et al. 2017). Using these tools, success or failure in finding the expected signatures is interpreted in terms of the mechanisms generating diversity in the studied clade. 
Despite a substantial research effort, the role and the effect of competition on diversification during evolutionary radiations remain controversial. Several studies indicate that competition within species can promote lineage splitting through disruptive selection on traits (i.e. ecological speciation; Dieckmann \& Doebeli 1999; Schluter 2001), but competition can also prevent diversification by reducing ecological opportunity and by driving species to extinction (Bailey et al. 2013; Rabosky 2013). How these contrasting effects of competition interact to shape traits and lineages macroevolutionary dynamics and the patterns observed in comparative datasets remains unclear (Meyer \& Kassen 2007; McPeek 2008; Bailey et al. 2013; Clarke et al. 2017). Intriguingly, while competition mechanistically links phenotypic evolution with speciation (via ecological character displacement, Doebeli \& Dieckmann 2000; Schluter 2001; Rundle \& Nosil 2005) and extinction (via competitive exclusion of phenotypically similar species, Sepkoski 1978), these processes have not been integrated in a unified macroevolutionary model (Weber et al. 2017).

In order to clarify the effect of competition on diversification dynamics and the patterns left on comparative data, we begin by developing an integrated lineage-based model, the Matching Competition Birth-Death model (MCBD), where traits evolve according to a matching competition process (Nuismer \& Harmon 2015; Drury et al. 2016) and influence speciation-extinction dynamics in a protracted birth-death process (Etienne \& Rosindell 2012). Next, we examine the effect of competition by simulating the MCBD model. Particularly, we investigate the conditions under which competition spurs species and phenotypic diversity, and generates the evolutionary rate declines expected from verbal adaptive radiation theory. Finally, we investigate the signatures left on comparative data and discuss our results.

\section{METHODS}

\section{The Model}

We model lineage diversification using a protracted speciation-extinction process (PBD, Etienne \& Rosindell 2012), an extension of the classical birth-death 
process (BD; Nee et al. 1994) that accounts for the fact that speciation takes time to complete (Etienne \& Rosindell 2012). Under the PBD, species produce "incipient species" at a rate $\lambda_{1}$ (speciation initiation) that become "good species" at a rate $\lambda_{2}$ (speciation completion). This modeling approach provides a convenient intermediate between simpler lineage-based BD models that cannot incorporate intra-specific processes such as competition-driven population divergence, and more mechanistic models from populations genetics (e.g. Slatkin, 1980) or adaptive dynamics (e.g. Doebeli \& Ispolatov 2017), which have the advantage of being based on first principles of drift, fitness, and response to selection, but generally do not make predictions that can be confronted to comparative phylogenetic data (but see Aguilée et al. 2018).

We consider two versions of the model: one with "asymmetric" speciation initiation (main text), where the ancestral good species persists as a good species and produces one daughter incipient species, and one with "symmetric" speciation initiation (Supplement Information), where the ancestral good species gives rise to two incipient lineages. Incipient lineages inherit trait values from their parent species, and do not give rise to new incipient species. Speciation completion is contingent upon the accumulation of phenotypic differences (Fig. 1a): the speciation completion rate of incipient species $i$ at time $t$ increases with the phenotypic distance with its parent good species (or sister incipient species) $j$ at this time:

$$
\lambda_{2 i}(t)=\tau_{0} e^{\beta\left(x_{i}(t)-x_{j}(t)\right)^{2}}
$$

where $\tau_{0}$ is the basal speciation completion rate, $x_{i}(t)$ and $x_{j}(t)$ are the trait values of lineages $i$ and $j$ at time $t$, and $\beta$ expresses the extent of the effect of trait differences on the speciation completion rate.

Lineages are driven to extinction if they fail to become sufficiently different from other competing lineages, according to the principles of limiting similarity and competitive exclusion (Fig. 1b). The extinction rate $\mu_{i}$ of a lineage $i$ (incipient or good) is a decaying function of the phenotypic distance with all other lineages at time $t$ :

$$
\mu_{i}=\alpha_{1} \mu_{0} e^{-\alpha_{1}\left(\sum_{j \neq i}\left(x_{i}(t)-x_{j}(t)\right)^{2}\right)}+\mu_{b g}
$$


where $\mu_{b g}$ is a background extinction rate, $\mu_{0}$ modulates the relative contribution of competition-related extinction with respect to $\mu_{b g}$, and $\alpha_{1}$ modulates the effect of trait differences on the extinction rate. $\mu_{b g}$ and $\mu_{0}$ can take distinct values for good and incipient species.

We model trait evolution using a modified version of the "matching competition" (MC) process described in Drury et al. (2018), which is based on a model derived by Nuismer \& Harmon (2015) from quantitative genetics first principles. The MC model represents character displacement, with competition between co-existing lineages driving phenotypic divergence (Fig. 1c). Competition-driven trait divergence facilitates speciation completion following Eq. 1, and reduces extinction following Eq. 2. We express the change of trait value $x$ on lineage $i$ after an infinitesimally small timestep $d t$ as:

$$
x_{i}(t+d t)=x_{i}(t)+m \alpha_{2}\left[\sum_{j \neq i}^{n} \operatorname{sign}\left(x_{i}(t)-x_{j}(t)\right) \times e^{-\alpha_{2}\left(x_{i}(t)-x_{j}(t)\right)^{2}}\right] d t+\delta
$$

where $\delta$, the Brownian Motion (BM), is a normally distributed random variable with mean 0 and variance $\sigma^{2} d t$, and $m$ is a scaling parameter modulating the contribution of the deterministic competition component with respect to the BM. The "sign" term determines the direction of the repulsion in trait space (i.e. species $i$ evolves towards higher trait values if $x_{i}>x_{j}$, and lower trait values if $x_{i}<x_{j}$ ), and $n$ is the number of lineages at time $t$. Finally, $\alpha_{2}$ modulates the effect of trait differences on trait evolution. When $\alpha_{2}=0$, trait evolution is purely neutral (i.e. following BM). Eq. 3 is equivalent to Eq. 1 from Drury et al. (2018), without the Ornstein-Uhlenbeck attraction to an optimum, and the $m$ parameter here equals $\frac{m_{D}}{\alpha_{2}}$ where $m_{D}$ is the $m$ parameter in Eq. 1 from Drury et al. (2018). This alternative parametrization allows us to evaluate the effect of competition on trait evolution by varying only the parameter $\alpha_{2}$. We consider two versions of the model: one with an unbounded trait space, and one with hard limits on trait space beyond which lineages cannot evolve (Clarke et al. 2017). Such hard limits can arise from physiological or genetic constraints. They also represent the 
idea of "ecological limits", that is the existence of a limited breadth of resources that puts a hard limit on the number of species a system can sustain (Rabosky 2009). Note that hard ecological limits are not required to produce ecological constraints: as soon as clades diversify and species start occupying niches, this imposes constraints on the trait values that emerging species can take.

Eqs. 2-3 implement a Gaussian functional form, a convenient and realistic approximation to model the effect of phenotypic distance in trait-driven models of competitive interactions (MacArthur \& Levins 1967; Dieckman \& Doebeli 1999). We kept a similar functional form (i.e. exponential) in Eq. 1 for consistency, but with the speciation completion rate increasing with phenotypic distance, ensuring that speciation occurs when large phenotypic differences are attained.

Finally, we allow all lineages to interact with one another, implying that they evolve in the same geographic area, a simplifying assumption but an arguably common feature of adaptive radiations (Stroud \& Losos 2016). We consider the case of a univariate trait, although it would be straightforward to extend Eqs. 1-3 to consider a multidimensional trait. The univariate case is the simplest, and could be a common scenario if radiations unfold along single, highly integrated phenotypic axes (e.g. lines of least evolutionary resistance; Schluter 1996).

\section{Simulations}

We implemented a time-discretization of the model in which we sequentially computed trait values and lineage speciation-extinction over many small time-steps. In order to control the crown age of the process, we started each simulation with one good and one incipient species (attributed equal trait values). We ran the simulations for $50 \mathrm{Ma}$, with time steps of $0.01 \mathrm{Ma}$. Each simulation produced a diversifying trajectory of lineages and traits through time (Fig. 1d,e). We followed these radiations by registering the traits' evolutionary history and time of all speciation/extinction events, which we used to build a phylogenetic tree of each process. This tree consisted of extinct lineages, and living good and incipient species (Fig. 1f). By pruning the latter 
and extinct lineages, we obtained a reconstructed tree and a vector of present-day trait values (Fig. 1f). Codes for running the simulations are available in RPANDA (function sim.MCBD; Morlon et al. 2016). We simulated 100 processes for each different parameter setting, discarding simulations that produced reconstructed trees with less than 5 tips or extinct radiations (although these rarely occurred).

In order to easily explore the effects of changing competition strength on diversification, we ran all our simulations with the same values for the effect of traitdriven competition on extinction $\left(\alpha_{1}\right)$ and trait evolution $\left(\alpha_{2}\right)$. We expect $\alpha_{1}$ and $\alpha_{2}$ to co-vary, as extinction and trait evolution are both driven by the same individual-level process, namely reduced survival of individuals experiencing strong competition (Dieckman \& Doebeli 1999). Thereafter we note $\alpha=\alpha_{1}=\alpha_{2}$, and refer to $\alpha$ as the competition strength. We ran a first set of simulations with no bounds in trait space. We set $\beta=0.6, m=0.2$ and varied competition strength $(\alpha=0-0.1)$. We ran simulations without and with extinction. For those with extinction, we set good and incipient species background extinction rates to $\mu_{\mathrm{bg} 1}=0.01$ and $\mu_{\mathrm{bg} 2}=0.016$, allowing for traitdependent extinction rates (competitive exclusion) by setting $\mu_{0}$ to 50 times $\mu_{b g}$ and $\mu_{b g} 2$ values, respectively. We set higher incipient extinction rates to account for potentially lower population sizes in incipient lineages. Population size will affect the background extinction rate through both stochastic demographic fluctuations (Lande, 1993) and genetic factors such as inbreeding depression (Frankham, 2005). It will also affect the competition-driven extinction rate as the response to directional selection (and the resulting effect on individuals' fitness if adaptation is too slow) depends on effective population size (Wright, 1931).

We fixed $\sigma^{2}=0.5$ to achieve a good balance between the stochastic and deterministic components of trait evolution. We then ran a second set of simulations with increasingly larger symmetric hard bounds on trait space around the root value and with extinction; fixing competition strength $(\alpha=0.04)$. All other parameter values were the same as in the unbounded simulations. These values were selected through preliminary exploration of the behavior of the model as to obtain biological realism. 
For example, ensuring trees of reasonable size for adaptive radiations (i.e. $<30$ tips when possible; Harmon et al. 2010) and extinction rates that were not too high (e.g. a maximum turnover rate of 0.4 in the most strongly competitive case).

\section{Model output}

To assess the effect of competition on species richness, trait diversity, and the dynamics of macroevolutionary rates under the MCBD model, we recorded for each radiation and at each time step species richness, phenotypic disparity, realized speciation, extinction and net diversification rates, speciation duration, and trait evolution rates (Appendix S1).

To investigate the footprint that competition leaves on phylogenetic trait data under the MCBD model, we applied a set of comparative tools commonly used for detecting adaptive radiations. For each reconstructed tree we computed the $\gamma$ statistic (Pybus \& Harvey 2000), a metric used to test for declines in diversification rates through time. Negative $\gamma$ values indicate a slowdown in speciation rate towards the present. We also compared the fit (using the AICc; Burnham \& Anderson 2004) of different models of diversification, representing: i) constant rates of speciation/extinction (with and without extinction), ii) time-dependent rates with linear/exponential dependences, or iii) diversity-dependent rates with linear dependency on speciation and constant or not extinction (Appendix S2). For each reconstructed tree and associated trait data, we estimated a measure of the phylogenetic signal ( $K$; Blomberg et al. 2003). $K=1$ is expected under BM and which $K$ values to expect under competition-driven radiations is not entirely clear (Appendix S2). We also compared the fit of commonly used models of phenotypic evolution representing: random (BM; Felsenstein 1985) or constrained (OU; Hansen 1997) evolution, exponentially-changing rates through time (ACDC; Blomberg et al. 2003), diversity-dependent rates (DD; Weir \& Mursleen 2013), and a model incorporating competitive interactions (MC; Drury et al. 2016). We also obtained reconstructed trees and associated $\gamma$ and $K$ values along the simulations. 
Comparative analyses were performed with the $\mathrm{R}$ packages RPANDA, geiger (Pennell et al. 2014), DDD (Etienne et al. 2012), and phytools (Revell 2012).

\section{RESULTS}

\section{Dynamics of speciation, extinction and species richness}

There were only few conditions under which competition increased the number of species in a clade (Fig. 2a,b \& Fig. 3a,b). Even in the absence of both trait limits and extinction, species richness was highest at intermediate (not at highest) competition strength (Fig. 2a,b). As expected from adaptive radiation theory, competition spurred diversification early in the radiation, but then generated fast declines in speciation rates (Fig. 2c). Indeed, competition accelerated speciation completion at the beginning of the radiation and then slowed it down toward the present (Fig. 2d). Hard trait limits were not required to obtain such patterns, as incipient species experienced the constraints imposed by coexisting species (Fig. S1a), which was sufficient to slow down speciation completion and limit species richness.

Competition had a strong effect on the intensity and temporal dynamics of extinction (Fig. 3c), which had cascading effects on other aspects of the radiation (compare Fig. 3 with Fig. 2). Extinction rates of good species were overall higher for stronger competition and increased nearly linearly with time as trait space became more packed (Fig. 3c). Extinction rates of incipient species were also higher for stronger competition. This erased the negative effect of competition on the time to complete speciation towards the present (compare Fig. 3d with Fig. 2d), as only fast diverging incipient lineages escaped extinction and became good species. Speciation rates therefore remained higher under strong competition throughout the radiation (Fig. 3c), even if they declined slightly through time as a result of frequent incipient species extinction (Fig. 3c). The combined effect of competition on extinction and speciation rates led to net diversification rates that were higher early in the radiation 
and declined faster later on (Fig. 3c). This resulted in a lower species richness (Fig. $3 a, b)$.

Finally, limiting trait space led to markedly fewer species compared to the unbounded case (Fig. 4a,b). This resulted from overall lower speciation rates caused by increasing failed speciation in narrower trait spaces, as there was almost no effect on speciation duration or extinction rates (Fig. 4c-d). Limiting trait space did not have a marked effect on temporal diversification dynamics: as in the unbounded case, speciation slowed down and extinction increased strongly, resulting in a net diversification rate slow down (Fig. 4c-d).

\section{Dynamics of trait disparity and evolution rates}

While competition did not necessarily increase clade size, it generated clades with high trait disparity (Fig. 2e \& 3e), unless this disparity was limited by hard trait bounds (Fig. 4e). Even low levels of competition lead to an exponential increase in trait disparity through time, compared to a linear increase in the absence of competition (Fig. 2e \& 3e). In unbounded trait spaces, even though competition generated all the ecological constraints on evolution mentioned above, this did not result in a slowdown in the accumulation of trait disparity. The accumulation of trait disparity only progressively slowed down in bounded trait spaces, and only once bounds were hit (which occurred increasingly late as boundaries got wider, Fig. 4e).

Contrary to what happened for species diversification dynamics, we rarely observed slowdowns in mean trait evolution rates (Fig. 2f, $3 \mathrm{f}$ and $4 \mathrm{f}$ ). There was an initial drop down very early in the radiation, in the period preceding the first speciation event, when trait space was empty and the only two existing lineages could freely evolve away from each other. After this short period, mean trait evolution rates were either slightly increasing (in unbounded radiations without extinction, Fig. 2f) or constant (in bounded and unbounded radiations with extinction, Fig. 3f \& 4f). These average rate patterns hide a strong heterogeneity across lineages (Fig. S2). Coherent with ideas of ecological opportunity and ecological limits, directional evolution in 
competition-driven radiations was faster in lineages near empty zones of trait space (i.e. edges in unbounded radiations), with near-stasis in the crowded, constrained middle (Fig. S2a,b). In the absence of extinction, this increase in evolutionary rate at edges overcompensated the decrease towards the middle, leading to the observed slight increase in mean rates (Fig. 2f \& S2a). Extinction erased this increase by moderating the competitive pressure experienced by lineages (Fig. 3f and S2b). Even in the presence of hard trait limits, when bounds were reached and diversity approximated equilibrium, mean rates did not decline (Fig. 4f \& S2c). Lineages near the bounds experienced slow rates as they could not evolve further, but intense competition drove many species extinct, constantly freeing up trait space. Overall, these cycles of extinction, branching and reoccupation of trait space leveled off evolutionary rate declines imposed by bounds.

\section{Patterns left in contemporary data}

$\gamma$ did not appear as a robust signal of competition (Fig. 5, first column). Without extinction, reconstructed trees showed increasingly negative $\gamma$ values with increasing competition strength (Fig. 5a), but neutral radiations also exhibited negative $\gamma$ values, indicating that at least part of this signal stemmed from the protracted nature of the speciation process. Moreover, $\gamma$ values between competitive and neutral radiations only began to diverge late in time (Fig. S3a). In the presence of extinction, $\gamma$ values increased rather than decreased with competition strength, as radiations with more intense competition experienced more extinctions (Fig. 5b). Finally, trees from bounded competition-driven radiations had $\gamma$ values that became less negative as trait space became narrower (Fig. 5c).

Diversification models were not particularly useful either for detecting competition-driven radiations (Fig. 5, second column). Without extinction, both neutral and competition-driven radiations produced an important proportion of reconstructed trees that best supported models with declining (mostly linearly) speciation rates. Extinction progressively erased the signal of diversification rate 
decline as competition increased: trees from neutral radiations indicated support for models of declining speciation and increasing extinction rates, while for competitiondriven radiations support increased toward models of constant speciation-extinction (Fig. 5b). Support for models with declining speciation and increasing extinction decreased further in the presence of bounds and as bound got narrower (Fig. 5c).

Phylogenetic signal $(K)$ in tip trait data provided a more consistent signature of competition (Fig. 5, third column). $K$ values were higher for competition-driven than for neutral radiations, indicating that as morphospace is progressively occupied, competition constrains closely related species to a limited portion of this space (Fig. S2). In the absence of bounds, the signature of competition provided by $K$ became stronger as the radiation proceeded (Fig. S3a,b). In bounded radiations, K was highest at the time when the bounds were hit, and then remained stable or slowly decreasing (Fig. S3c). $K$ therefore decreased with increasingly tight trait spaces, but remained generally higher than under neutral radiations (Fig. 5c, the only exception occurred in the most constrained trait space).

The other consistent signature of competition was the statistical support for the MC model (Fig. 5, last column), which is noteworthy as it is different in several aspects from the generating model (Appendix S2). Trait model fitting generally recovered the MC model as the best for all competition-driven radiations, while the BM model was recovered for most neutral radiations (Fig. 5a,b). Support for the MC model was however progressively lost as bounds got tighter (Fig. 5c). Models with decreasing rates of evolution (e.g. diversity or time dependent rates) were never selected over MC. When we nevertheless examined the recovered rate change parameter for the ACDC model in competition-driven radiations, we found that it was generally negative (representing a model with declining rates), the only exception being tightly bounded radiations where this parameter was positive (Fig. S4).

\section{DISCUSSION}


We investigated the macroevolutionary consequences of interspecific competition during radiations, and the signal left on contemporary data under several scenarios. We found four main results: first, competition-driven radiations promote trait diversity, but not necessarily species richness; second, competition-driven radiations show declines in speciation and net diversification rates, even in unbounded trait spaces, but not in trait evolution rates, even in bounded trait spaces; third, testing for early burst patterns of species and/or trait diversification in comparative data does not provide a good test of adaptive radiations; fourth, phylogenetic signal in trait data and support for matching competition models may provide promising alternatives for detecting adaptive radiations.

It has been suggested that much of life's diversity, both in terms of species number and body form, originated as adaptive radiations (Simpson 1953). Whether this is the case or not, here we find that competition-driven radiations tend to generate phenotypic diversity, but not higher species richness than neutral radiations. While competition-driven character displacement increases speciation rates at the beginning of clades' history, either the ecological constraints in trait space imposed by competing species or competitive exclusion ("Darwinian extinction", Sepkoski 1978; Webb 2003; Pfennig \& Pfennig 2005) limit this diversity-generating process once trait space is packed.

Another general expectation of verbal theories of adaptive radiation is that there are "early bursts" of both speciation and trait evolution, with rates that are highest at early stages of diversification, declining later as competition for ecological space increases (Simpson 1953; Schluter 2000). We did observe early burst patterns of speciation: rates are initially high as fast trait divergence in an empty ecological space leads to fast speciation, declining afterwards. In the absence of extinction, the decline is driven by a "duration" control of speciation (Dynesius \& Jansson 2014): speciation takes more time to complete as incipient lineages have more difficulty diverging ecologically from their parent species in an increasingly packed morphological space. In the presence of extinction, the decline is instead driven by increasing extinction of 
incipient lineages, as in "ephemeral speciation" models (Stanley 1979; Futuyma 2010; Rosenblum et al. 2012). Bounds in trait space and saturation of ecological space exacerbate competition-driven declines in diversification rates, but are not required: the constraints in phenotypic space imposed by competing lineages is sufficient by itself to slow down diversification as radiations proceed. Although radiating in multidimensional trait spaces might alleviate these constraints (Doebeli \& Ispolatov 2017), if species mostly diversify along single or a few phenotypic axes (e.g. lines of least evolutionary resistance; Schluter 1996), the described scenario might be common and invocation of contentious hard ecological limits to diversity not needed, favoring "damped" diversification explanations instead (Cornell 2013, Harmon \& Harrison 2015).

Conversely, we did not observe early burst patterns of trait evolution in competitive radiations, even in bounded trait spaces. Mean rates tended to remain constant or even slightly increased. As lineages diversify trait space becomes more packed and competitive pressure increases (this occurs even in multidimensional phenotypic spaces; Doebeli \& Ispolatov 2017). In radiations far from (or without) ecological limits and with no extinction, fast competition-driven evolution at edges of occupied trait space overcompensates the effect of the constraints imposed by trait space packing in its central region; this even results in slight increases in mean trait evolution rates. Some empirical studies have indeed found evidence for higher rates at extremes of occupied morphospace (e.g. Cooper \& Purvis 2009) or increasing evolutionary rates towards the present in adaptive traits (e.g. Venditti et al. 2011; Hopkins \& Smith 2015). When increased competition leads to frequent extinctions, these open up trait/niche space, which reduces the competitive pressure to evolve at edges and the constraints imposed by trait space packing in its central region, resulting in nearly constant mean trait evolution rates. These results are consistent with recent paleontological studies that did not find evidence for trait evolution rate declines in adaptive macroevolutionary diversification (Hopkins \& Smith 2015). Hence, the idea that mean trait evolution rates will decline during adaptive radiations because of 
increased competitive constraints on morphospace occupation might not necessarily hold; instead, we suggest that under some conditions character displacement and high lineage turnover fostered by increased competitive pressure may balance the effect of niche packing. Empirical studies are needed to clarify the potential generality of this process.

As expected (even for constant-rate neutral evolution; Foote 1996), in bounded trait spaces trait disparity reaches its maximum when boundaries are reached. However, competition does not necessarily lead lineages to trait boundaries early in a radiation unless the bounds are small compared to niche width: character displacement pushes species away in trait space, but it does so just enough to escape competition, so that it takes time before lineages reach the boundaries of trait space. Thus, our model predicts that relatively early disparity peaks may only be observed in adaptive radiations that are either very old compared to the moment where they hit bounds, or characterized by a tightly constrained trait space where significant species diversification would be difficult. Hence, if early disparity peaks are repetitively observed across species-rich clades irrespective of their age, as has been observed in the fossil record (Hughes et al. 2013), this suggests that the traits considered evolve in such constrained morphological spaces (Foote 1996; Gavrilets 1999), but that these traits are not the ones that are involved in competitive interactions and niche partitioning (if such interactions and partitioning occur).

Taken together, our results show a decoupling between rates that are often thought to be coupled. In particular, rates of trait evolution can remain constant (or even increase) despite decreasing diversification rates. And in adaptive radiations that reached ecomorphological limits, rates of trait evolution can remain constant despite disparity reaching a plateau, similarly to the "fly in a tube" effect (Felice et al. 2018). These two decoupling are both linked to the continuous reoccupation of vacated trait space after extinctions.

Biologists have searched for early burst patterns in comparative data as a signal of adaptive radiations (Harmon et al. 2003, 2010; Moen \& Morlon 2014). Paradoxically, 
despite not producing declines in trait evolution rates, fits of the ACDC model to our simulated competition-driven radiations infer a decelerating rate process, except in tightly bounded trait spaces where an increasing rate process is inferred (Fig. S4). These later results add to a growing literature suggesting that temporal trends found when fitting an ACDC model to extant trait data should not be used to draw conclusions about the process itself (Revell et al. 2008; Uyeda et al. 2015). Our analyses also confirm that early bursts of diversification may be difficult to discriminate from an apparent decline stemming from the protracted nature of speciation (Etienne \& Rosindell 2012). Additionally, we corroborate that extinction erases the signature of declining rates in reconstructed trees (Rabosky \& Lovette 2008b; Liow et al 2010). Thus, commonly observed slowdowns in real phylogenies (McPeek 2008; Phillimore \& Price 2008; Morlon et al. 2010) might not necessarily be indicative of ecological modes of diversification but of other mechanisms (Moen \& Morlon 2014). Our results suggest that increasing extinction rate could be another mark of adaptive radiations (as proposed in previous studies, e.g. McPeek 2008; Rabosky \& Lovette 2008a; Aguilée et al. 2018), but that this signal is not picked up by common likelihood-based models of diversification.

While searching for early bursts in comparative data does not seem the most reliable way to detect competition-driven radiations, our results suggest that alternative signals could be exploited. The two clear signatures are a strong phylogenetic signal (as measured by Blomberg's K) and a greater likelihood of phenotypic models incorporating competitive interactions (Drury et al. 2016). That competition leads to high phylogenetic signal (indicating that closely related species are more similar than expected) is perhaps unanticipated, as character displacement will tend to increase differences between two competing species (Pfennig \& Pfennig 2009). Conversely, here we show (similarly to Clarke et al. 2017) that tree wide competition, while increasing overall disparity, tends to limit the morphospace occupied by subclades, leading to a strongly structured trait distribution in which species tend to occupy the same trait regions as their ancestors (e.g. niche 
conservatism; Harmon et al. 2003; Losos 2008). The only scenarios under which competition-driven radiations do not generate a high phylogenetic signal are scenarios with tight trait bounds (Fig. 5c; Revell et al. 2008) that produce low species diversity. Those are also the only exceptions to the otherwise widespread support for matching competition models (Drury et al. 2016). Support for models incorporating competitive interactions is even more informative than phylogenetic signal alone. Indeed, while a declining rate unrelated to competition would also generate phylogenetic signal (Revell et al. 2008), trait evolution models can distinguish these two scenarios because they induce a different morphospace occupation: gaps in trait space under declining rates, and more even distribution under competition (Clarke et al. 2017). It would be interesting to test if novel statistics that capture finer scale variation in a trait's distribution than Blomberg's K (e.g. Lewitus et al. in review) are also able to distinguish these two scenarios, as well as to check if phylogenetic signal remains a feature of adaptive radiations in multidimensional trait space (Harvey \& Rambaut 2007, Doebeli \& Ispolatov 2017). Likewise, it would be useful to assess the robustness of the support for Drury et al.'s MC models (2016) with a variety of competition-driven radiation scenarios, as it could be argued that our generating model resembles the MC inference tool. In addition to a strong phylogenetic signal and support for the matching competition model, competition-driven radiations can generate heterogeneous trait evolution rates (and also most likely diversification rates), with higher rates for species at the extremes of occupied morphospace. This pattern could potentially be exploited with recent comparative methods that allow estimating branch-specific rates (Venditti et al. 2011; Rabosky 2014; Maliet et al. 2019).

As with any modelling approach, we made a series of simplifying assumptions that may impact our results. In particular, population-level dynamics or landscape features may affect macroevolutionary outcomes (Aguilée et al. 2018). Nonetheless, we were able to investigate in a formal framework some fundamental ideas on adaptive radiations that clarify the macroevolutionary consequences of competition and will hopefully help design more precise and powerful tools to investigate them. 


\section{AKNOWLEDGMENTS}

We thank J. P. Drury, M. Manceau, J. Clavel and E. Lewitus for their help and useful comments, and more generally members of the Morlon lab. We also thank J. Rosindell and three anonymous reviewers whose comments helped to greatly improve the quality of this manuscript. This work was funded by grant PANDA from the European Research Council (ERC-CoG) awarded to HM.

\section{REFERENCES}

Aguilée, R., Gascuel, F., Lambert, A. \& Ferriere, R. (2018). Clade diversification dynamics and the biotic and abiotic controls of speciation and extinction rates. Nat. Commun., 9, 1-13.

Bailey, S.F., Dettman, J.R., Rainey, P.B. \& Kassen, R. (2013). Competition both drives and impedes diversification in a model adaptive radiation. Proc. R. Soc. B Biol. Sci., 280.

Blomberg, S.P., Garland Jr., T. \& Ives, A.R. (2003). Testing for phylogenetic signal in comparative data: behavioral traits are more labile. Evolution, 57, 717-745.

Burnham, K.P. \& Anderson, D.R. (2004). Multimodel inference: Understanding AIC and BIC in model selection. Sociol. Methods Res., 33, 261-304.

Clarke, M., Thomas, G.H. \& Freckleton, R.P. (2017). Trait Evolution in Adaptive Radiations: Modeling and Measuring Interspecific Competition on Phylogenies. Am. Nat., 189, 121-137.

Cornell, H.V. (2013). Is regional species diversity bounded or unbounded? Biol. Rev., $88,140-165$.

Cooper, N., Purvis, A. (2009). What factors shape rates of phenotypic evolution? A comparative study of cranial morphology of four mammalian clades. J. Evol. Biol., $22,1024-1035$.

Dieckmann, U. \& Doebeli, M.O. (1999). On the origin of species by sympatric speciation. Nature, 400, 354-357. 
Doebeli, M. \& Dieckmann, U. (2000). Evolutionary Branching and Sympatric Speciation Caused by Different Types of Ecological Interactions. Am. Nat., 156, S77-S101.

Doebeli, M., \& Ispolatov, I. (2017). Diversity and coevolutionary dynamics in highdimensional phenotype spaces. Am. Nat., 189, 105-120.

Drury, J., Clavel, J., Manceau, M. \& Morlon, H. (2016). Estimating the effect of competition on trait evolution using maximum likelihood inference. Syst. Biol., 65, 700-710.

Drury, J.P., Grether, G.F., Garland Jr., T. \& Morlon, H. (2018). An assessment of phylogenetic tools for analyzing the interplay between interspecific interactions and phenotypic evolution. Syst. Biol., 67, 413-427.

Dynesius, M. \& Jansson, R. (2014). Persistence of within-species lineages: A neglected control of speciation rates. Evolution, 68, 923-934.

Edwards, A.W.F. \& Cavalli-Sforza, L.L. (1964). Reconstruction of evolutionary trees. Phenetic Phylogenetic Classif., 6, 67-76.

Etienne, R.S., Haegeman, B., Stadler, T., Aze, T., Pearson, P.N., Purvis, A., et al. (2012). Diversity-dependence brings molecular phylogenies closer to agreement with the fossil record. Proc. R. Soc. B Biol. Sci., 279, 1300-1309.

Etienne, R.S. \& Rosindell, J. (2012). Prolonging the past counteracts the pull of the present: Protracted speciation can explain observed slowdowns in diversification. Syst. Biol., 61, 204-213.

Felice, R.N., Randau, M., \& Goswami, A. (2018). A fly in a tube: Macroevolutionary expectations for integrated phenotypes. Evolution, 72, 2580-2594.

Felsenstein, J. (1985). Phylogenies and the Comparative Method. Am. Nat., 125, 1-15.

Foote, M. (1996). Models of morphological diversification. Evol. Paleobiol. Univ. Chicago Press. Chicago, 62-86.

Frankham, R. (2005). Genetics and extinction. Biol. Cons., 126, 131-140.

Futuyma, D.J. (1998). Evolutionary Biology. Sinauer Associates, Sunderland, MA.

Futuyma, D.J. (2010). Evolutionary constraint and ecological consequences. Evolution, 
$64,1865-1884$.

Gavrilets, S. (1999). Dynamics of clade diversification on the morphological hypercube. Proc. R. Soc. B Biol. Sci., 266, 817-824.

Gavrilets, S. \& Losos, J.B. (2009). Adaptive Radiation: Contrasting Theory with Data. Science, 323, 732-737.

Hansen, T.F. (1997). Stabilizing selection and the comparative analysis of adaptation. Evolution, 51, 1341-1351.

Harmon, L.J. \& Harrison, S. (2015). Species diversity is dynamic and unbounded at local and continental scales. Am. Nat., 185, 584-593.

Harmon, L.J., Losos, J.B., Davies, J.T., Gillespie, R.G., Gittleman, J.L., Jennings, B.W., et al. (2010). Early bursts of body size and shape evolution are rare in comparative data. Evolution, 64, 2385-2396.

Harmon, L.J., Schulte II, J.A., Larson, A. \& Losos, J.B. (2003). Tempo and Mode of Evotutionary Radiation in Iguanian Lizards. Science, 301, 961-964.

Hopkins, M.J. \& Smith, A.B. (2015). Dynamic evolutionary change in post-Paleozoic echinoids and the importance of scale when interpreting changes in rates of evolution. Proc. Natl. Acad. Sci., 201418153.

Hughes, M., Gerber, S. \& Wills, M.A. (2013). Clades reach highest morphological disparity early in their evolution. Proc. Natl. Acad. Sci., 110, 13875-13879.

Lande, R. (1993). Risks of population extinction from demographic and environmental stochasticity and random catastrophes. Am. Nat. 142, 911-927.

Lewitus, E., Aristide, L. \& Morlon, H. Characterizing and comparing phylogenetic trait data from their normalized Laplacian spectrum. bioRxiv 654087 [preprint]. May 31, 2019 (cited 2019 August 10). Available from: https://doi.org/10.1101/654087

Liow, L.H., Quental, T.B. \& Marshall, C.R. (2010). When can decreasing diversification rates be detected with molecular phylogenies and the fossil record?. Syst. Biol., 59, 646-659.

Liow, L.H., Reitan, T. \& Harnik, P.G. (2015). Ecological interactions on macroevolutionary time scales: Clams and brachiopods are more than ships that 
pass in the night. Ecol. Lett., 18, 1030-1039.

Losos, J.B. (2008). Phylogenetic niche conservatism, phylogenetic signal and the relationship between phylogenetic relatedness and ecological similarity among species. Ecol. Lett., 11, 995-1003.

MacArthur, R., \& Levins, R. (1967). The limiting similarity, convergence, and divergence of coexisting species. Am. Nat., 101, 377-385.

Mahler, D.L., Revell, L.J., Glor, R.E. \& Losos, J.B. (2010). Ecological opportunity and the rate of morphological evolution in the diversification of greater Antillean anoles. Evolution, 64, 2731-2745.

Maliet, O., Hartig, F. \& Morlon, H. (2019). A model with many small shifts for estimating species-specific diversification rates. Nature Ecol. Evol., 3,1086-1092.

Manceau, M., Lambert, A. \& Morlon, H. (2017). A Unifying Comparative Phylogenetic Framework Including Traits Coevolving Across Interacting Lineages. Syst. Biol., $66,551-568$.

McPeek, M.A. (2008). The Ecological Dynamics of Clade Diversification and Community Assembly. Am. Nat., 172, E270-E284.

Meyer, J.R. \& Kassen, R. (2007). The effects of competition and predation on diversification in a model adaptive radiation. Nature, 446, 432-435.

Moen, D. \& Morlon, H. (2014). Why does diversification slow down? Trends Ecol. Evol., 29, 190-197.

Morlon, H., Lewitus, E., Condamine, F.L., Manceau, M., Clavel, J. \& Drury, J. (2016). RPANDA: An R package for macroevolutionary analyses on phylogenetic trees. Methods Ecol. Evol., 7, 589-597.

Morlon, H., Potts, M.D. \& Plotkin, J.B. (2010). Inferring the dynamics of diversification: a coalescent approach. PLoS Biol., 8, e1000493.

Nee, S., May, R.M. \& Harvey, P.H. (1994). The reconstructed evolutionary process. Phil. Trans. R. Soc. Lond. B, 344, 305-311.

Nuismer, S.L. \& Harmon, L.J. (2015). Predicting rates of interspecific interaction from phylogenetic trees. Ecol. Lett., 18, 17-27. 
Pagel, M. (1999). Inferring the historical patterns of biological evolution. Nature, 401, 877-884.

Pennell, M.W., Eastman, J.M., Slater, G.J., Brown, J.W., Uyeda, J.C., FitzJohn, R.G., et al. (2014). geiger v2. 0: an expanded suite of methods for fitting macroevolutionary models to phylogenetic trees. Bioinformatics, 30, 2216-2218.

Pennell, M.W. \& Harmon, L.J. (2013). An integrative view of phylogenetic comparative methods: Connections to population genetics, community ecology, and paleobiology. Ann. N. Y. Acad. Sci., 1289, 90-105.

Pfennig, K. \& Pfennig, D. (2009). Character displacement: ecological and reproductive responses to a common evolutionary problem. Q. Rev. Biol., 84, 253-276.

Pfennig, K.S. \& Pfennig, D.W. (2005). Character displacement as the "best of a bad situation": fitness trade-offs resulting from selection to minimize resource and mate competition. Evolution, 59, 2200-2208.

Phillimore, A.B. \& Price, T.D. (2008). Density-dependent cladogenesis in birds. PLoS Biol., 6, e71.

Pybus, O.G. \& Harvey, P.H. (2000). Testing macro-evolutionary models using incomplete molecular phylogenies. Proc. R. Soc. London B Biol. Sci., 267, 2267-2272.

Rabosky, D.L. (2009). Ecological limits and diversification rate: alternative paradigms to explain the variation in species richness among clades and regions. Ecol. Lett., $12,735-743$.

Rabosky, D.L. (2013). Diversity-Dependence, ecological speciation, and the role of competition in macroevolution. Annu. Rev. Ecol. Evol. Syst., 44, 481-502.

Rabosky, D.L. (2014). Automatic detection of key innovations, rate shifts, and diversity-dependence on phylogenetic trees. PLoS One, 9, e89543.

Rabosky, D.L. \& Lovette, I.J. (2008a). Density-dependent diversification in North American wood warblers. Proc. R. Soc. B Biol. Sci., 275, 2363-2371.

Rabosky, D.L. \& Lovette, I.J. (2008b). Explosive evolutionary radiations: Decreasing speciation or increasing extinction through time? Evolution, 62, 1866-1875.

Revell, L.J. (2012). phytools: an R package for phylogenetic comparative biology (and 
other things). Methods Ecol. Evol., 3, 217-223.

Revell, L.J., Harmon, L.J. \& Collar, D.C. (2008). Phylogenetic signal, evolutionary process, and rate. Syst. Biol., 57, 591-601.

Rosenblum, E.B., Sarver, B.A.J., Brown, J.W., Des Roches, S., Hardwick, K.M., Hether, T.D., et al. (2012). Goldilocks meets Santa Rosalia: An ephemeral speciation model explains patterns of diversification across time scales. Evol. Biol., 39, 255-261.

Rundle, H.D. \& Nosil, P. (2005). Ecological speciation. Ecol. Lett., 8, 336-352.

Schluter, D. (1996). Adaptive radiation along genetic lines of least resistance. Evolution, 50,1766-1774.

Schluter, D. (2000). The ecology of adaptive radiation. OUP Oxford.

Schluter, D. (2001). Ecology and the origin of species. Trends Ecol. Evol., 16, 372-380.

Sepkoski Jr., J.J. (1978). A kinetic model of Phanerozoic taxonomic diversity I. Analysis of marine orders. Paleobiology, 4, 223-251.

Sepkoski Jr, J.J. (1996). Competition in macroevolution: the double wedge revisited. Evol. Paleobiol., 211-255.

Silvestro, D., Antonelli, A., Salamin, N. \& Quental, T.B. (2015). The role of clade competition in the diversification of North American canids. Proc. Natl. Acad. Sci., $112,8684-8689$.

Simpson, G.G. (1953). The Majors Features of Evolution. Columbia University Press, New York.

Slatkin, M. (1980). Ecological character displacement. Ecology, 61, 163-177.

Soulebeau, A., Aubriot, X., Gaudeul, M., Rouhan, G., Hennequin, S., Haevermans, T., et al. (2015). The hypothesis of adaptive radiation in evolutionary biology: hard facts about a hazy concept. Org. Divers. Evol., 15, 747-761.

Stanley, S.M. (1979). Macroevolution, pattern and process. Johns Hopkins University Press.

Stroud, J.T. \& Losos, J.B. (2016). Ecological opportunity and adaptive radiation. Annu. Rev. Ecol. Evol. Syst., 47, 507-532.

Uyeda, J.C., Caetano, D.S. \& Pennell, M.W. (2015). Comparative analysis of principal 
components can be misleading. Syst. Biol., 64, 677-689.

Venditti, C., Meade, A. \& Pagel, M. (2011). Multiple routes to mammalian diversity. Nature, 479, 393-396.

Vermeij, G.J. (2013). On Escalation. Annu. Rev. Earth Planet. Sci., 41, 1-19.

Webb, C. (2003). A complete classification of Darwinian extinction in ecological interactions. Am. Nat., 161, 181-205.

Weber, M.G., Wagner, C.E., Best, R.J., Harmon, L.J. \& Matthews, B. (2017). Evolution in a community context: On integrating ecological interactions and macroevolution. Trends Ecol. Evol., 32, 291-304.

Weir, J.T. \& Mursleen, S. (2013). Diversity-dependent cladogenesis and trait evolution in the adaptive radiation of the auks (Aves: Alcidae). Evolution, 67, 403-416.

Wright, S. (1931). Evolution in Mendelian populations. Genetics, 16, 97.

\section{SUPPORTING INFORMATION}

Additional Supporting Information may be downloaded via the online version of this article at Wiley Online Library (www.ecologyletters.com).

\section{FIGURE LEGENDS}

\section{Figure 1 - An integrated phylogenetic model of traits and species diversification.}

The speciation completion rate of a lineage depends on the distance with its parental species (A). The extinction rate (B) and the competition-driven component of trait evolution (C) are plotted as a function of the distance between two competing lineages. The $\alpha$ parameters control competition strength. Higher $\alpha$ values model a scenario where more similar phenotypes compete more strongly, implying a strong association between trait values and resource use. Conversely, a scenario where the evolving trait is less important for resource allocation is modeled by lower $\alpha$ values, as competition is less intense and less related to phenotypic differences among lineages. (D, E) 
Simulations produce a diversifying trajectory of species and traits through time, and we study the effect of competition by varying $\alpha$ values. (D) shows an example radiation under competitive evolution, and (E) under purely neutral evolution. Lineages in orange are incipient species. (F) From a simulation we obtain a tree describing the full diversification process (first panel), from which we derive the fossil tree (second panel) and the reconstructed tree with trait values at tips (third panel).

Figure 2 - Effect of competition on diversification patterns in an unbounded trait space and no extinction. We performed 100 simulations for each $\alpha$ value. All other parameters were fixed (see methods). (A) Mean species richness (log scale) through time. (B) Final species richness (reconstructed tree size). (C, D) Realized mean speciation rates and speciation duration through time. (E) Mean trait disparity. (F) Mean trait evolution rate. Colored lines in $(C, D, F)$ are a smoothed function fitted to the colored dots (1 Ma bins) for clearer representation. Notice the higher stochasticity at the beginning of the simulations given the fewer lineages present.

Figure 3 - Effect of competition on diversification patterns in an unbounded trait space with competitive exclusion. (A) Mean species richness (log scale) through time. (B) Final species richness (reconstructed tree size). (C) Realized mean speciation rates, good species extinction rates, and net diversification rates (speciation minus extinction). (D) Speciation duration. (E) Mean trait disparity. (F) Mean trait evolution rate.

Figure 4 - Effect of competition on diversification patterns with bounds on trait space and competitive exclusion. Competition strength was fixed at an intermediate value $(\alpha=0.04)$. Trait/ecological space was increasingly larger (higher width). (A) Mean species richness (log scale) through time. (B) Final species richness (reconstructed tree size). C) Realized mean speciation rates, good species extinction 
rates, and net diversification rates (speciation minus extinction). (D) Speciation duration. (E) Mean trait disparity. (F) Mean trait evolution rate.

Figure 5 - Patterns recovered on reconstructed trees and trait tip data using phylogenetic comparative methods. Results for simulated radiations without (A) and with competitive exclusion (B) and no bounds, and with competitive exclusion and bounds (C). From left to right: $\gamma$ statistic for tree shape; species diversification model fit (proportion of trees showing support for a given birth-death model, where B/D indicate speciation/extinction, and cst, lin and exp indicate constant, linearly or exponentially time-changing rates); phylogenetic signal $(K)$ in trait data; and phenotypic evolution model fit (proportion of trees showing support for each of the following models: Matching Competition (MC), single optimum Ornstein-Uhlenbeck $(\mathrm{OU})$, exponentially increasing or decreasing rates through time (ACDC), Brownian motion (BM), and exponential (DDe) or linear (DDl) diversity-dependent rates). See Appendix S2 for details. 
A

B

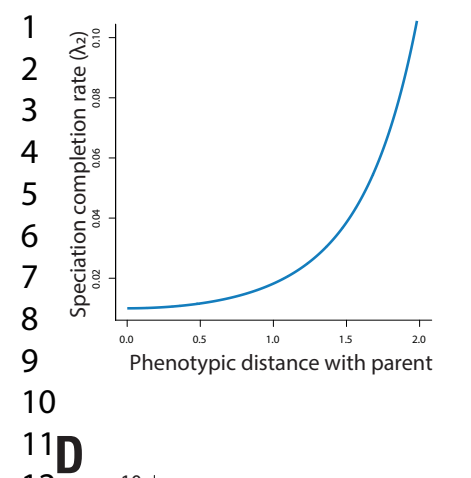

12

13

14

15

16

17

18

19

20

21

22

$23 \mathrm{~F}$

24

25

26

27

28

29

30

31

32

33

34

35

36

37

38

39

40

41

42

43

44

45

46

47

48

49

50

51

52

53

54

55

56

57

58

59

60

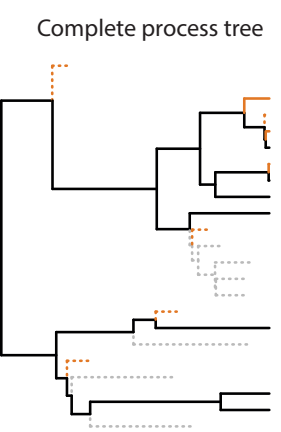

... Extinct incipient species

Extinct good species

Extant incipient species

Page 28 of 32

C
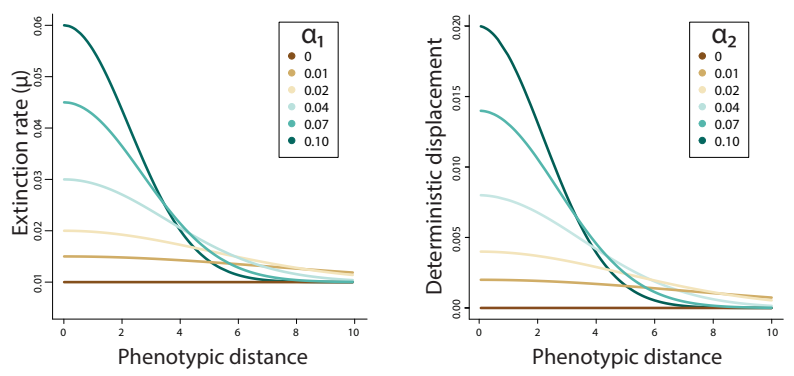

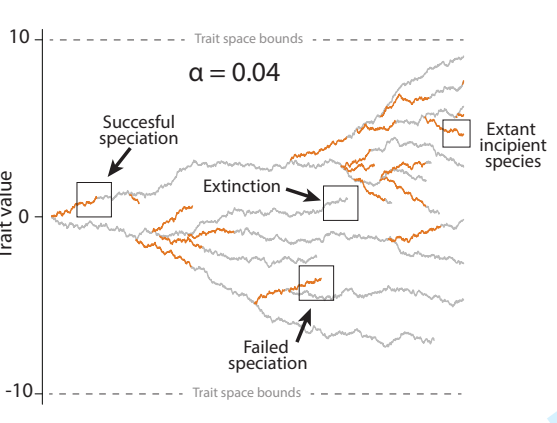

E

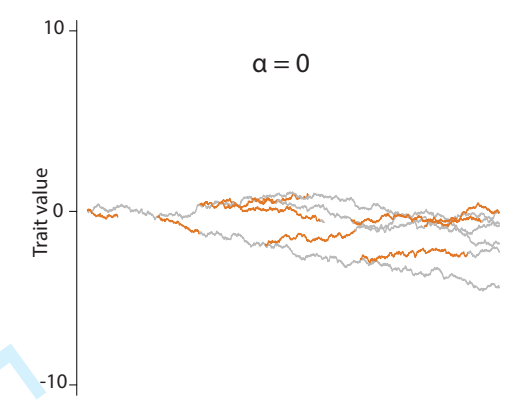

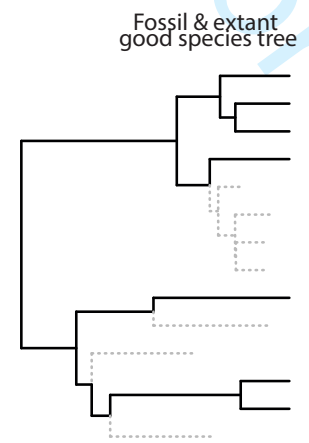

Reconstructed good species tree
$\&$ trait values

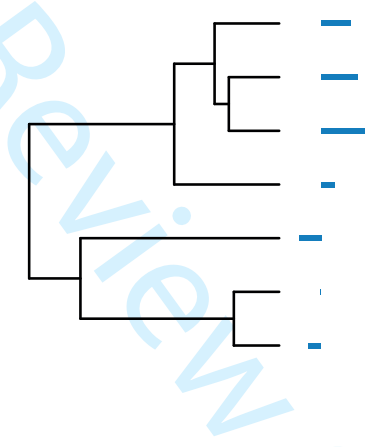


Page 29 of 32

Ecology Letters

A

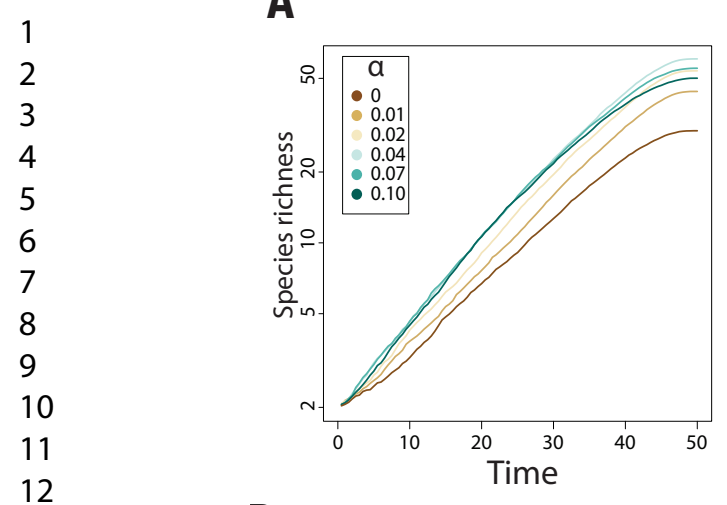

13 D

14

15

16

17

18

19

20

21

22

23

24

25

26

27

28

29

30

31

32

33

34

35

36

37

38

39

40

41

42

43

44

45

46

47

48

49

50

51

52

53

54

55

56

57

58

59

60
B

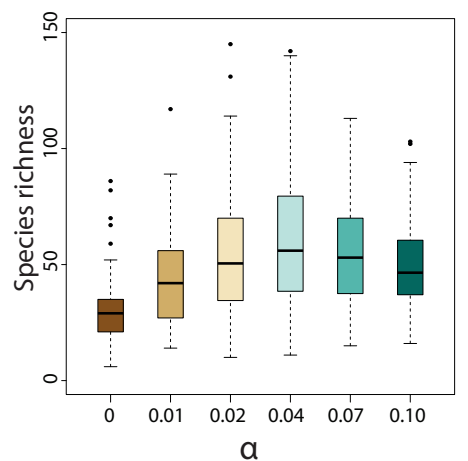

E

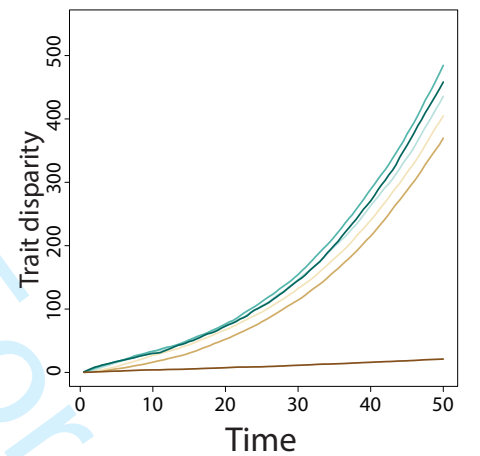

C

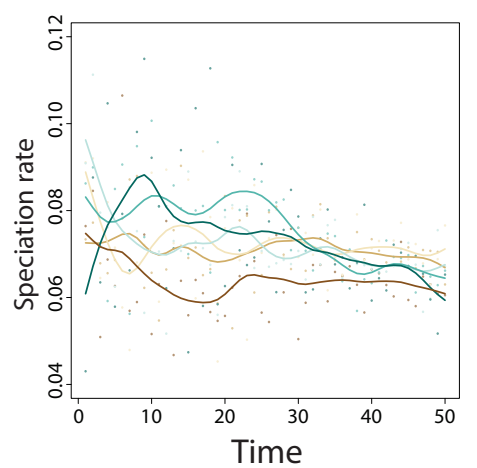

F

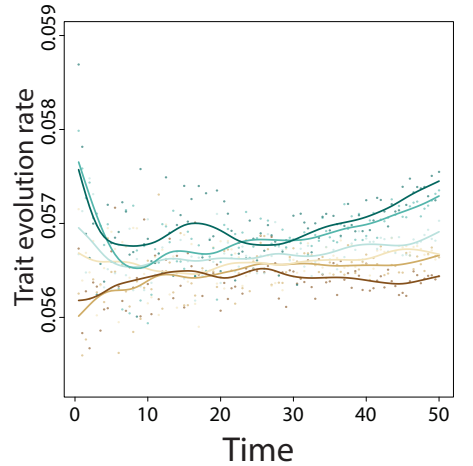


Ecology Letters

Page 30 of 32

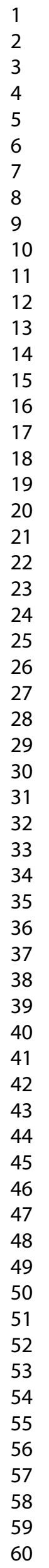

B
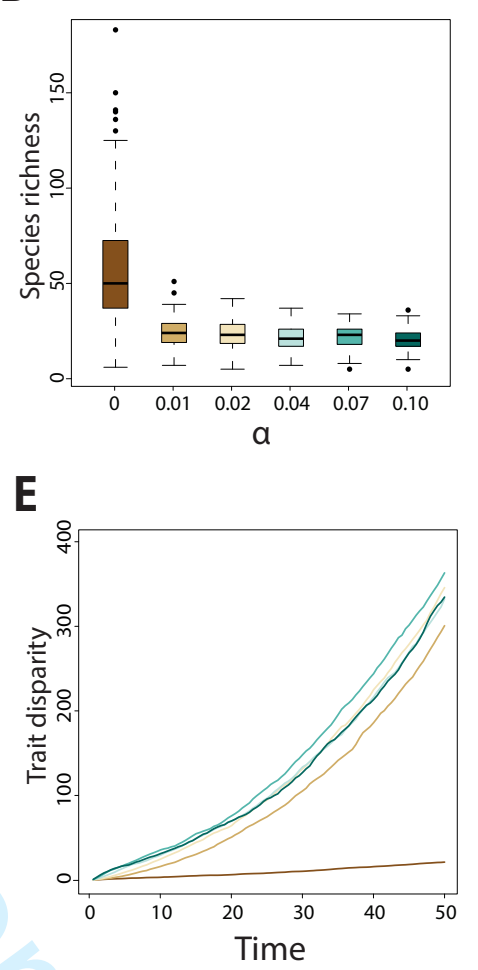

C

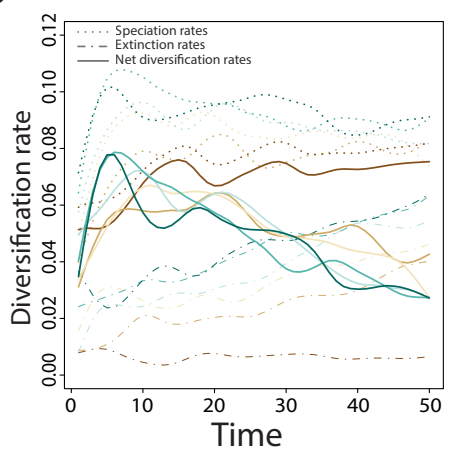

$\mathbf{F}$

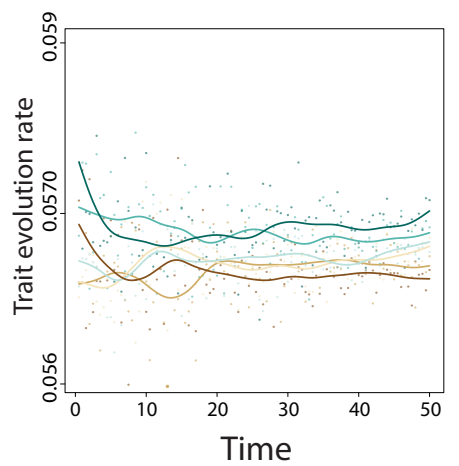

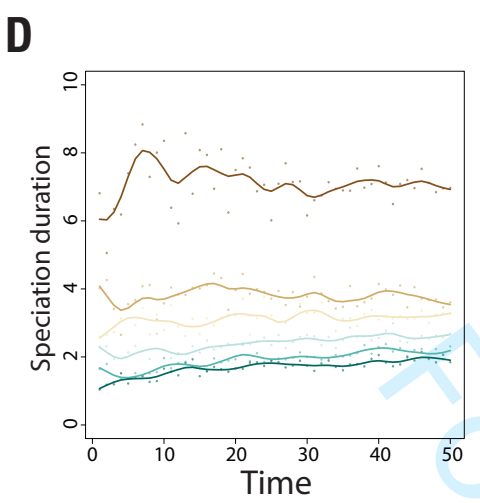


Page 31 of 32

Ecology Letters

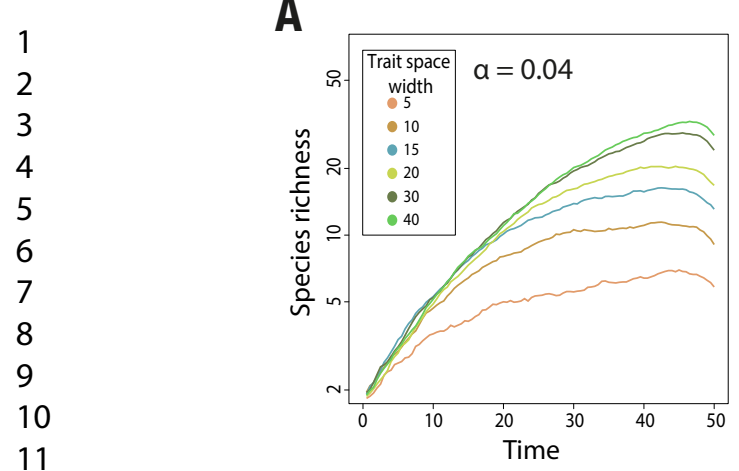

12

13

14

15

16

17

18

19

20

21

22

23

24

25

26

27

28

29

30

31

32

33

34

35

36

37

38

39

40

41

42

43

44

45

46

47

48

49

50

51

52

53

54

55

56

57

58

59

60
A

B

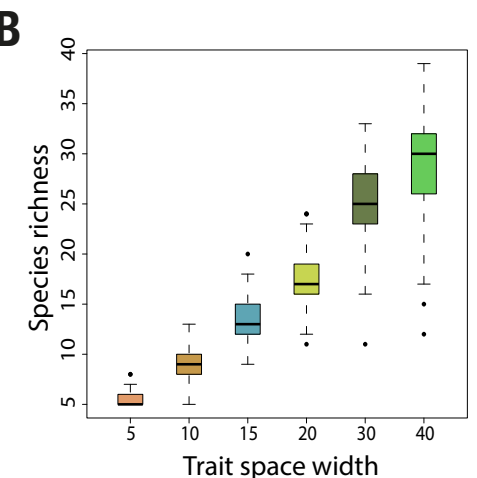

E

D

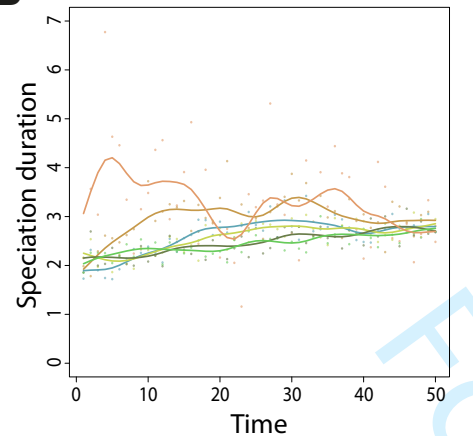

C

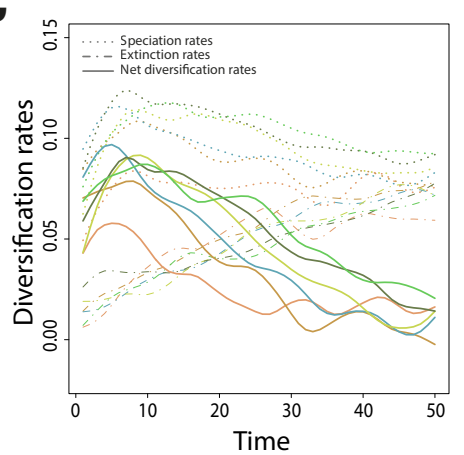

$\mathbf{F}$

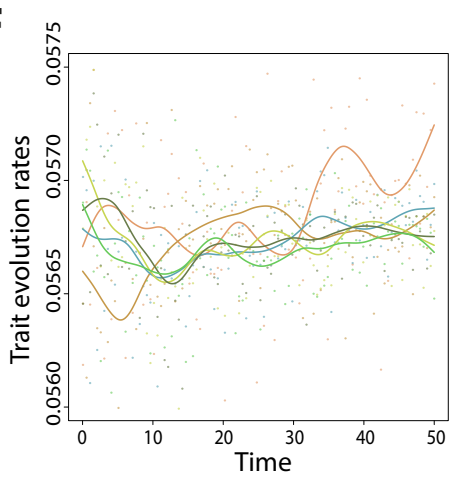


